\title{
OPTICAL AND NEAR INFRARED OBSERVATIONS OF POST-AGB STARS
}

\author{
T.FUJII \\ Dept. of Astronomy, Univ. of Tokyo \\ fujii@mtk.ioa.s.u-tokyo.ac.jp \\ T.ONO \\ Nishi-Harima Astronomical Observatory \\ ono@nhao.go.jp \\ Y. NAKADA \\ Kiso Observatory, Univ. of Tokyo \\ nakada@kiso.ioa.s.u-tokyo.ac.jp \\ AND \\ M.PARTHASARATHY \\ Indian Institute of Astrophysics, Bangalore \\ partha@iiap.ernet.in
}

The post-AGB stars rapidly change their spectral energy distribution on their way from the asymptotic giant branch(AGB) to planetary nebula stage. They are generally surrounded by cool and extended dust shells emitting largely in the infrared wavelengths.

A selected sample of the post-AGB candidates were observed using the CCD and IR camera at Kiso and Nishi-Harima Astronomical Observatory. They were classified based on their broad band spectra from B to K' band and their evolutionary stages have been discussed using the simple model of the dust shell around a star.

The light variation of some of these objects were monitored and their light curves are also presented. 